\title{
Effect of colestimide on the concentrations of polychlorinated dibenzo-p-dioxins, polychlorinated dizenzofurans, and polychlorinated biphenyls in blood of Yusho patients
}

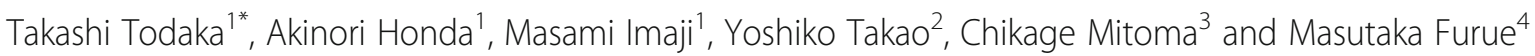

\begin{abstract}
Background: Oral colestimide was reported to lower the concentration of PCDDs, PCDFs, and PCB in the blood of humans. A pilot study showed that the arithmetic mean total TEQ concentrations of PCDDs, PCDFs, and PCBs in the blood of subjects after the trial decreased approximately $20 \%$ compared to pre-trial levels, suggesting that colestimide could decrease human dioxin levels. We designed the current clinical trial study based on this information. In this study, we examined whether colestimide could reduce the individual congener concentrations of PCDDs, PCDFs, and PCBs in the blood of Yusho patients.

Methods: Out of the 36 Yusho patients who participated in the clinical trial, 26 patients self-administered colestimide $3 \mathrm{~g} /$ day orally for 6 months. The concentrations of PCDDs, PCDFs and PCBs in the blood of 26 Yusho patients before the trial were compared with those after the trial.

Results: The arithmetic mean total TEQ concentrations of PCDDs, PCDFs, non-ortho PCBs, and mono-ortho PCBs in the blood of the 26 Yusho patients before and after the clinical trial were 42-303 (mean: 130, median: 120) and 43-283 (mean: 132, median: 118) pg TEQ/g lipid, respectively. The sums of the concentrations of 58 PCB congeners measured in the blood of Yusho patients before and after the trial were 321-2643 (mean: 957, median: 872) and 286-2007 (mean: 975, median: 806) ng/g lipid, respectively, indicating that the concentrations of PCDDs, PCDFs, and PCBs after the trial were almost the same as those before the trial. Among congeners of PCDDs, PCDFs, dioxin-like PCBs, and non-dioxin-like PCBs, most congeners of these compounds did not show a statistically significant decrease after the trial.
\end{abstract}

Conclusion: Colestimide may not be beneficial in reducing the high blood levels of dioxin-like compounds in Yusho patients.

Keywords: PCDDs, PCDFs, PCBs, Yusho, Colestimide, Blood concentration

\section{Background}

The 1968 Yusho poisoning accident affected over 1800 people in western Japan [1]. Since the Yusho outbreak, the National Study Group for the Therapy of Yusho has carried out medical care and health examinations of patients affected [2]. In 2001, the measurement of

\footnotetext{
*Correspondence: todaka@klsc.or.jp

${ }^{1}$ Kitakyushu Life Science Center, Public Interest Incorporated Foundation, Nakabarushinmachi 1-4, Tobata-ku, Kitakyushu-shi, Fukuoka 804-0003, Japan Full list of author information is available at the end of the article
}

PCDDs, PCDFs, and non-ortho PCBs in the blood became possible using small amounts of blood collected from participants during annual medical examinations [3-5]. We have measured the concentrations of PCDDs, PCDFs, and dioxin-like PCBs in the blood collected from Yusho patients in medical health examinations since 2002 [6-8]. Moreover, we have conducted a congener-specific analysis of non-dioxin-like PCBs in the blood of these patients since 2004 $[9,10]$. 
Based on these results, we previously reported that Yusho patients continue to have higher concentrations of PCDFs in their blood than unaffected people, and that concentration of PCDFs in the blood is significantly correlated with the intensity of Yusho symptoms $[11,12]$.

Development of effective therapy to reduce the concentrations of PCDDs, PCDFs, and PCBs in the blood of Yusho patients could improve the health care of these patients. With regard to promoting the excretion of lipophilic contaminants stored in the human body, several studies of dietary supplements such as cholestyramine, mineral oil, hexadecane, and dietary fiber have been reported using laboratory animals [13-16]. In addition, another study reported the enhancing effect of non-absorbable lipid substitute olestra on fecal excretion of PCDDs, PCDFs, and PCBs in the human body $[17,18]$. Our study group previously conducted a clinical trial to reduce the concentrations of PCDDs, PCDFs, and PCB in the blood of Yusho patients using cholestyramine and rice bran fiber $[19,20]$. However, beneficial clinical effects could not be confirmed due to the short trial period.

Colestimide, a 2-methylimidozarol-epichlorohydrin polymer, is widely used to lower serum cholesterol levels in Japan. Recently, oral colestimide was reported to lower the concentration of PCDDs, PCDFs, and PCB in the blood of humans $[21,22]$. A pilot study showed that the arithmetic mean total TEQ concentrations of PCDDs, PCDFs, and PCBs in the blood of subjects after the trial decreased approximately $20 \%$ compared to pretrial levels, suggesting that colestimide could decrease human dioxin levels $[21,22]$. We designed the current clinical trial study based on this information. In this study, we examined whether colestimide could reduce the individual congener concentrations of PCDDs, PCDFs, and PCBs in the blood of Yusho patients.

\section{Methods \\ Sampling}

The trial protocol was approved by the institutional ethics committee of Kyusyu University Hospital. Patients who fulfilled the diagnostic criteria for Yusho established by the National Study Group for the Therapy of Yusho were eligible for this study. Patients were recruited at explanatory meetings conducted in Fukuoka and Nagasaki Prefectures. 50 Yusho patients were enrolled in this clinical trial, and 14 patients refused to participate. The remaining 36 patients participated in the trial. Informed consent was obtained for study participation. The patients self-administrated colestimide $3 \mathrm{~g} /$ day orally for 6 months. Out of the 36 Yusho patients who participated in the clinical trial, 26 patients completed the trial. The 26 patients ranged in age from 60 to 87 years (mean: 72.9, median: 72.5). Among the 26 patients, there were 13 men (age range 60-87 years; mean: 73.1, median: 74.0) and 13 women (age range 61-81 years; mean: 72.8, median: 72.0). The blood samples examined in this study were collected between April 4, 2008 and July 15, 2009. After collection, the blood samples were stored at $4{ }^{\circ} \mathrm{C}$ until analyses.

\section{Materials}

Native congeners of PCDDs, PCDFs, dioxin-like PCBs, and non-dioxin-like PCBs were purchased from Wellington Laboratories (Guelph, Canada). $\left[{ }^{13} \mathrm{C}_{12}\right]$-congeners of PCDDs, PCDFs, dioxin-like PCBs, and non-dioxin-like PCBs as internal standards, were also purchased from Wellington Laboratories. An active carbon column was prepared as follows: active carbon was purchased from Nacalai Tesque (Kyoto, Japan), refluxed 3 times with toluene for $1 \mathrm{~h}$, and dried in vacuum, after which $500 \mathrm{mg}$ of the active carbon was mixed with $500 \mathrm{~g}$ of anhydrous sodium sulfate (Wako Pure Chemical Industries, Ltd., Tokyo, Japan). A silver nitrate/silica gel was purchased from Wako Pure Chemical Industries, Ltd. All reagents and solvents used in this experiment were of the analytic grade of dioxin that is commercially available.

\section{Analysis of PCDDs, PCDFs, and PCBs}

The extraction and purification of PCDDs, PCDFs, dioxinlike PCBs, and non-dioxin-like PCBs from blood samples were performed using a previously reported method $[5,9]$. Concentrations of PCDDs, PCDFs, and dioxin-like PCBs and concentrations of 58 non-dioxin-like PCB congeners were determined by a previously reported method $[5,9]$.

\section{Quality control}

To evaluate the accuracy and reliability of the analysis of PCDDs, PCDFs, dioxin-like PCBs, and non-dioxinlike PCBs, our laboratory prepared human blood samples and conducted quality control studies of the analysis of PCDDs, PCDFs, and dioxin-like PCBs in 2007, 2009, 2011, and 2013 and non-dioxin-like PCBs in 2008, 2010, 2012, and 2014. Each quality control study involved the participation of various laboratories that perform measurements for these compounds in human blood in Japan. In each quality control study, our results were compared with those of participating laboratories, and tests confirmed that the average variation among values obtained by each organization performing the analysis was all within $10 \%$. These results indicated that our laboratory's analytical methods regarding PCDDs, PCDFs, dioxin-like PCBs, and non-dioxin-like PCBs in human blood provided accurate results. 
Table 1 Effect of colestimide on the individual congener concentrations of PCDDs, PCDFs, and dioxin-like PCBs in the blood of Yusho patients

\begin{tabular}{|c|c|c|c|c|c|c|c|c|c|c|c|}
\hline \multirow[t]{3}{*}{ Congeners } & \multicolumn{10}{|c|}{ Concentration (pg/g lipid) } & \multirow[t]{3}{*}{$p$ Values } \\
\hline & \multicolumn{5}{|c|}{ Before the clinical trial } & \multicolumn{5}{|c|}{ After the clinical trial } & \\
\hline & Mean & Median & SD & Minimum & Maximum & Mean & Median & SD & Minimum & Maximum & \\
\hline 2,3,7,8-TetraCDD & 1.8 & 1.7 & 0.9 & 0.5 & 4.0 & 2.0 & 1.8 & 1.2 & 0.5 & 4.7 & 0.083 \\
\hline 1,2,3,7,8-PentaCDD & 14 & 14 & 4.9 & 6.6 & 23 & 14 & 12 & 6.1 & 6.1 & 27 & 0.675 \\
\hline 1,2,3,4,7,8-HexaCDD & 3.1 & 3.0 & 1.7 & 1.0 & 7.1 & 3.3 & 3.2 & 1.7 & 1.0 & 6.9 & 0.053 \\
\hline 1,2,3,6,7,8-HexaCDD & 62 & 53 & 36 & 16 & 183 & 63 & 55 & 34 & 15 & 164 & 0.258 \\
\hline 1,2,3,7,8,9-HexaCDD & 5.5 & 4.5 & 5.2 & 2.1 & 29 & 5.7 & 3.9 & 6.0 & 1.0 & 31 & 0.770 \\
\hline 1,2,3,4,6,7,8-HeptaCDD & 55 & 47 & 25 & 21 & 113 & 52 & 43 & 27 & 20 & 143 & 0.137 \\
\hline OctaCDD & 699 & 606 & 281 & 323 & 326 & 670 & 543 & 309 & 305 & 1610 & 0.118 \\
\hline Total PCDD & 841 & 739 & 315 & 413 & 1525 & 811 & 688 & 346 & 382 & 1850 & 0.144 \\
\hline 2,3,7,8-TetraCDF & 2.8 & 2.7 & 1.3 & 0.5 & 5.5 & 2.7 & 2.6 & 1.4 & 0.5 & 5.8 & 0.427 \\
\hline 1,2,3,7,8-PentaCDF & 1.3 & 1.1 & 0.9 & 0.5 & 3.5 & 1.5 & 1.2 & 1.1 & 0.5 & 4.4 & 0.554 \\
\hline 2,3,4,7,8-PentaCDF & 241 & 191 & 158 & 48 & 636 & 242 & 205 & 158 & 49 & 613 & 0.732 \\
\hline 1,2,3,4,7,8-HexaCDF & 64 & 51 & 56 & 7.8 & 227 & 64 & 52 & 56 & 8.1 & 207 & 0.990 \\
\hline 1,2,3,6,7,8-HexaCDF & 26 & 21 & 19 & 6.2 & 86 & 26 & 22 & 19 & 5.2 & 74 & 0.534 \\
\hline 2,3,4,6,7,8-HexaCDF & 1.2 & 1.0 & 0.7 & 1.0 & 3.4 & 1.2 & 1.0 & 0.6 & 1.0 & 3.4 & 1.000 \\
\hline 1,2,3,7,8,9-HexaCDF & ND & & & & & ND & & & & & \\
\hline 1,2,3,4,6,7,8-HeptaCDF & 2.2 & 1.0 & 1.5 & 1.0 & 6.5 & 2.3 & 1.0 & 1.8 & 1.0 & 7.8 & 0.820 \\
\hline 1,2,3,4,7,8,9-HeptaCDF & ND & & & & & ND & & & & & \\
\hline OctaCDF & ND & & & & & ND & & & & & \\
\hline Total PCDF & 342 & 280 & 229 & 71 & 963 & 344 & 292 & 230 & 71 & 890 & 0.732 \\
\hline 33'4'4'-TriCB(\#77) & 6.9 & 5.0 & 3.7 & 5.0 & 16 & 8.9 & 7.5 & 4.3 & 5.0 & 20 & 0.016 \\
\hline 344'5-TriCB(\#81) & 5.3 & 5.0 & 1.4 & 5.0 & 12 & 5.7 & 5.0 & 2.4 & 5.0 & 15 & 0.180 \\
\hline 33'44'5-PentaCB(\#126) & 129 & 100 & 81 & 30 & 391 & 131 & 96 & 85 & 34 & 356 & 0.770 \\
\hline 33'44'55'-HexaCB(169) & 279 & 250 & 144 & 104 & 678 & 293 & 280 & 129 & 114 & 585 & 0.101 \\
\hline Total Non-ortho PCBs & 420 & 382 & 178 & 183 & 906 & 439 & 406 & 166 & 196 & 789 & 0.078 \\
\hline 233'44'-PentaCB(\#105) & 4454 & 3145 & 3555 & 1206 & 13788 & 4581 & 3236 & 3714 & 5.0 & 15228 & 0.501 \\
\hline 2344'5-PentaCB(\#114) & 2800 & 2365 & 1688 & 5.0 & 7194 & 2997 & 2681 & 1699 & 5.0 & 6987 & 0.118 \\
\hline 23'44'5-PentaCB(\#118) & 21718 & 16568 & 17601 & 5.0 & 75475 & 21050 & 15412 & 14335 & 4575 & 57260 & 0.990 \\
\hline 2'344'5-PentaCB(\#123) & 304 & 228 & 273 & 5.0 & 1239 & 312 & 214 & 237 & 5.0 & 898 & 0.581 \\
\hline 233'44'5-НеxaCB(\#156) & 50472 & 32661 & 46375 & 13079 & 195017 & 51038 & 30741 & 43667 & 9528 & 180163 & 0.517 \\
\hline 233'44'5'-HexaCB(\#157) & 13157 & 8088 & 13150 & 3390 & 53954 & 12747 & 7644 & 11520 & 2332 & 46994 & 0.990 \\
\hline 23'44'55'-НехаCB(\#167) & 4834 & 4243 & 3373 & 5.0 & 16863 & 4610 & 4265 & 2422 & 985 & 10481 & 0.770 \\
\hline 233'44'55'-HeptaCB(\#189) & 7385 & 5100 & 5888 & 1664 & 24429 & 7398 & 5397 & 5323 & 1730 & 22434 & 0.829 \\
\hline Total Mono-ortho PCBs & 105125 & 83472 & 66740 & 40066 & 293077 & 104734 & 93659 & 59308 & 34746 & 267273 & 0.829 \\
\hline TEQ from PCDDs & 24 & 24 & 7.9 & 11 & 43 & 24 & 24 & 9.2 & 11 & 42 & 0.809 \\
\hline TEQ from PCDFs & 82 & 63 & 54 & 16 & 223 & 82 & 68 & 54 & 16 & 211 & 0.534 \\
\hline TEQ from PCDDs/PCDFs & 106 & 83 & 60 & 27 & 265 & 107 & 87 & 61 & 28 & 249 & 0.790 \\
\hline TEQ from non-ortho PCBs & 21 & 20 & 9.8 & 7.4 & 54 & 22 & 20 & 9.7 & 7.8 & 47 & 0.485 \\
\hline TEQ from mono-ortho PCBs & 3.2 & 2.5 & 2.0 & 1.2 & 8.8 & 3.1 & 2.8 & 1.8 & 1.0 & 8.0 & 0.829 \\
\hline TEQ from dioxin-like PCBs & 24 & 22 & 11 & 9.0 & 60 & 25 & 23 & 11 & 9.1 & 51 & 0.603 \\
\hline Total TEQ & 130 & 120 & 65 & 42 & 303 & 132 & 117 & 65 & 43 & 283 & 0.869 \\
\hline
\end{tabular}


Table 2 Effect of colestimide on the individual congener concentrations of non-dioxin-like PCBs in the blood of Yusho patients

\begin{tabular}{|c|c|c|c|c|c|c|c|c|c|c|c|}
\hline \multirow[t]{3}{*}{ IUPAC\# } & \multicolumn{10}{|c|}{ Concentration (pg/g lipid) } & \multirow[t]{3}{*}{$p$ Values } \\
\hline & \multicolumn{5}{|c|}{ Before the clinical trial } & \multicolumn{5}{|c|}{ After the clinical trial } & \\
\hline & Mean & Median & SD & Minimum & Maximum & Mean & Median & SD & Minimum & Maximum & \\
\hline TriCB-28 & 1644 & 1449 & 866 & 324 & 3809 & 1837 & 1866 & 1226 & 5 & 6187 & 0.025 \\
\hline TriCB-29 & 20 & 12 & 18 & 5 & 72 & 20 & 5 & 23 & 5 & 99 & 0.845 \\
\hline TriCB-37 & 128 & 5 & 245 & 5 & 847 & 73 & 5 & 165 & 5 & 698 & 0.112 \\
\hline TeteraCB-44 & 348 & 248 & 523 & 5 & 2841 & 415 & 324 & 415 & 107 & 2261 & 0.034 \\
\hline TeteraCB-47/48 & 525 & 359 & 437 & 117 & 1769 & 640 & 471 & 715 & 121 & 3659 & 0.049 \\
\hline TeteraCB-49 & 295 & 179 & 409 & 44 & 1679 & 344 & 216 & 576 & 5 & 3070 & 0.101 \\
\hline TeteraCB-52/69 & 956 & 780 & 836 & 294 & 4572 & 1060 & 860 & 745 & 368 & 3896 & 0.052 \\
\hline TeteraCB-56/60 & 442 & 306 & 344 & 5 & 1412 & 489 & 284 & 577 & 104 & 3010 & 0.889 \\
\hline TeteraCB-63 & 116 & 117 & 65 & 5 & 280 & 140 & 118 & 69 & 5 & 360 & 0.382 \\
\hline TeteraCB-66 & 2118 & 1520 & 1507 & 586 & 5853 & 2181 & 1536 & 1691 & 613 & 8475 & 0.551 \\
\hline TeteraCB-70 & 362 & 130 & 807 & 13 & 3375 & 418 & 143 & 1308 & 55 & 6817 & 0.280 \\
\hline TeteraCB-71 & 37 & 11 & 56 & 5 & 238 & 126 & 5 & 490 & 5 & 2524 & 0.586 \\
\hline TeteraCB-74 & 14823 & 12720 & 9202 & 3830 & 41089 & 14505 & 11875 & 9068 & 2973 & 35194 & 0.770 \\
\hline PentaCB-85 & 247 & 139 & 335 & 5 & 1592 & 205 & 138 & 218 & 5 & 1086 & 0.657 \\
\hline PentaCB-87 & 812 & 797 & 448 & 5 & 1716 & 747 & 697 & 442 & 5 & 2059 & 0.183 \\
\hline PentaCB-92 & 719 & 571 & 482 & 5 & 2402 & 752 & 669 & 455 & 5 & 2264 & 0.412 \\
\hline PentaCB-93/95/98 & 727 & 637 & 439 & 5 & 1964 & 1003 & 746 & 1165 & 326 & 6428 & 0.258 \\
\hline PentaCB-99 & 23623 & 19114 & 17453 & 4240 & 90685 & 24873 & 23328 & 16634 & 4308 & 82151 & 0.182 \\
\hline PentaCB-101 & 1931 & 1534 & 1234 & 5 & 5667 & 2337 & 1959 & 1481 & 600 & 6915 & 0.174 \\
\hline PentaCB-107/108 & 963 & 785 & 707 & 5 & 3340 & 961 & 819 & 584 & 5 & 2435 & 0.166 \\
\hline PentaCB-110 & 339 & 242 & 325 & 5 & 1451 & 332 & 298 & 268 & 5 & 1428 & 0.638 \\
\hline PentaCB-117 & 1911 & 1466 & 1813 & 435 & 7951 & 1722 & 1306 & 1642 & 5 & 6579 & 0.280 \\
\hline НехаCВ-128 & 925 & 685 & 660 & 5 & 3099 & 949 & 678 & 775 & 5 & 3899 & 0.443 \\
\hline HexaCB-130 & 7065 & 5603 & 5780 & 2080 & 25122 & 7238 & 5886 & 5578 & 1913 & 25258 & 0.568 \\
\hline HexaCB-132 & 399 & 326 & 252 & 5 & 1125 & 445 & 397 & 282 & 5 & 1134 & 0.143 \\
\hline HexaCB-134 & 25 & 5 & 50 & 5 & 183 & 35 & 5 & 47 & 5 & 168 & 0.203 \\
\hline HexaCB-135 & 419 & 342 & 318 & 5 & 1577 & 485 & 330 & 403 & 5 & 1587 & 0.382 \\
\hline HexaCB-137 & 10565 & 7132 & 9066 & 2996 & 41244 & 10646 & 7786 & 8734 & 2336 & 39991 & 0.889 \\
\hline HexaCB-138 & 96984 & 89163 & 52967 & 25546 & 240863 & 97685 & 84306 & 53897 & 23381 & 244647 & 0.990 \\
\hline HexaCB-139/149 & 635 & 452 & 619 & 15 & 2404 & 615 & 292 & 696 & 5 & 2303 & 0.568 \\
\hline HexaCB-141 & 328 & 255 & 246 & 5 & 1044 & 340 & 282 & 287 & 5 & 1169 & 0.716 \\
\hline НехаСВ-146 & 32968 & 34220 & 16346 & 11603 & 83149 & 35211 & 31688 & 16262 & 9839 & 68936 & 0.086 \\
\hline НехаCB-147 & 724 & 567 & 463 & 5 & 1678 & 768 & 622 & 519 & 5 & 1806 & 0.527 \\
\hline HexaCB-151 & 1329 & 981 & 880 & 428 & 3402 & 1349 & 1008 & 1098 & 5 & 4265 & 0.258 \\
\hline HexaCB-153 & 200929 & 184176 & 106109 & 73832 & 516088 & 206380 & 180663 & 109234 & 59314 & 458743 & 0.501 \\
\hline НехаCB-163/164 & 48797 & 47157 & 25168 & 17426 & 113577 & 49567 & 47872 & 22738 & 15767 & 88552 & 0.694 \\
\hline НехаCB-165 & ND & & & & & ND & & & & & \\
\hline HeptaCB-170 & 69704 & 60801 & 39523 & 16403 & 194289 & 69053 & 62645 & 37071 & 9946 & 142922 & 0.829 \\
\hline HeptaCB-172 & 9947 & 9273 & 5381 & 2768 & 27207 & 10156 & 10553 & 5277 & 5 & 20365 & 0.354 \\
\hline HeptaCB-177 & 15845 & 14513 & 9212 & 4504 & 39642 & 16359 & 14681 & 9932 & 2598 & 40496 & 0.424 \\
\hline HeptaCB-178 & 15885 & 13134 & 11788 & 5 & 56211 & 17209 & 13068 & 11123 & 3710 & 42945 & 0.012 \\
\hline HeptaCB-179 & 281 & 182 & 296 & 5 & 1110 & 357 & 202 & 384 & 5 & 1463 & 0.005 \\
\hline
\end{tabular}


Table 2 Effect of colestimide on the individual congener concentrations of non-dioxin-like PCBs in the blood of Yusho patients (Continued)

\begin{tabular}{|c|c|c|c|c|c|c|c|c|c|c|c|}
\hline HeptaCB-180 & 205779 & 201272 & 136971 & 50473 & 703408 & 203297 & 188508 & 121684 & 32744 & 490934 & 0.970 \\
\hline HeptaCB-181 & 553 & 292 & 671 & 5 & 2776 & 581 & 296 & 716 & 5 & 2806 & 0.264 \\
\hline HeptaCB-182/187 & 76063 & 60684 & 61028 & 14834 & 270253 & 81845 & 61046 & 63293 & 14093 & 238587 & 0.019 \\
\hline HeptaCB-183 & 16843 & 14980 & 12005 & 4733 & 45012 & 17864 & 15557 & 13292 & 3756 & 55788 & 0.182 \\
\hline HeptaCB-191 & 3078 & 2922 & 2008 & 5 & 8667 & 2907 & 2762 & 1688 & 805 & 7561 & 0.280 \\
\hline OctaCB-194 & 31774 & 32293 & 22776 & 5 & 116675 & 32519 & 32465 & 19427 & 8258 & 84219 & 0.304 \\
\hline OctaCB-195 & 7832 & 6835 & 5594 & 5 & 26180 & 7929 & 7156 & 4776 & 1879 & 19564 & 0.381 \\
\hline OctaCB-196/203 & 17107 & 15138 & 11346 & 5 & 55263 & 16821 & 15312 & 9331 & 4346 & 38617 & 0.869 \\
\hline OctaCB-198/201 & 14771 & 12536 & 11520 & 5 & 56995 & 14368 & 11829 & 8673 & 4251 & 34263 & 0.534 \\
\hline OctaCB-200 & 659 & 485 & 607 & 5 & 2350 & 705 & 593 & 609 & 5 & 2449 & 0.083 \\
\hline OctaCB-202 & 5432 & 3893 & 4532 & 5 & 22569 & 5298 & 3892 & 3307 & 1509 & 12550 & 0.258 \\
\hline OctaCB-205 & 977 & 898 & 633 & 5 & 2795 & 969 & 912 & 456 & 289 & 1949 & 0.770 \\
\hline NonaCB-206 & 5049 & 4561 & 2829 & 1502 & 14874 & 4891 & 4639 & 2446 & 5 & 10726 & 0.657 \\
\hline NonaCB-207 & 922 & 755 & 572 & 5 & 2501 & 911 & 792 & 522 & 5 & 1905 & 0.326 \\
\hline NonaCB-208 & 1877 & 1731 & 1209 & 5 & 6338 & 1827 & 1723 & 953 & 5 & 4559 & 0.778 \\
\hline DecaCB-209 & 1857 & 1598 & 890 & 893 & 5115 & 1900 & 1946 & 730 & 837 & 4005 & 0.200 \\
\hline Total TrCBs & 1792 & 1471 & 999 & 334 & 3921 & 1931 & 1917 & 1198 & 708 & 6225 & 0.182 \\
\hline Total TeCBs & 20023 & 17013 & 11246 & 6548 & 50619 & 20318 & 16713 & 11351 & 5981 & 43194 & 0.424 \\
\hline Total PeCBs & 31271 & 26027 & 19945 & 7137 & 102693 & 32932 & 31001 & 19076 & 7680 & 93210 & 0.228 \\
\hline Total HxCBs & 402098 & 373141 & 196792 & 152976 & 892316 & 411718 & 358344 & 198664 & 131539 & 769040 & 0.675 \\
\hline Total HpCBs & 413979 & 401285 & 265364 & 105126 & 1341206 & 419627 & 378119 & 250647 & 80774 & 971840 & 0.620 \\
\hline Total OcCBs & 78553 & 77549 & 55605 & 35 & 281931 & 78608 & 77101 & 44919 & 21477 & 186948 & 0.409 \\
\hline Total NoCBs & 7849 & 6439 & 4416 & 2355 & 23712 & 7629 & 7224 & 3832 & 15 & 17159 & 0.869 \\
\hline Total DeCBs & 1857 & 1598 & 890 & 893 & 5115 & 1900 & 1946 & 730 & 837 & 4005 & 0.200 \\
\hline Total PCBs & 957422 & 871523 & 520304 & 320807 & 2642555 & 974664 & 806289 & 495089 & 286088 & 2006817 & 0.585 \\
\hline
\end{tabular}

ND (less than the detection limit) values introduced to half values of the detection limit and calculated the TEQ concentrations

$S D$ standard deviation, $C B$ chlorinated biphenyl

\section{Data analysis}

To estimate the TEQ concentrations, we introduced ND (less than the detection limit) values to half values of the detection limit and calculated based on the TEF values proposed by the WHO [23]. The statistical analysis was conducted using Wilcoxon signed-rank test in the software programs from Statistics Package for Social Sciences (version 22; IBM Armonk, NY, USA). Significant probabilities ( $p$ values) were calculated for the respective number of samples analyzed.

\section{Results}

The objective of the present study was to evaluate the effectiveness of colestimide on the individual congener concentrations of PCDDs, PCDFs, and PCBs in blood of Yusho patients. Of the 36 Yusho patients who began the trial, 9 patients stopped administrating colestimide due to serious adverse effects, constipation or abdominal distension. Of the 27 remaining patients, we failed to collect a posttreatment blood sample from one patient due to cancellation of hospital visit. The individual congener concentrations of PCDDs, PCDFs and PCBs in the blood of 26 Yusho patients before the trial were compared with those after the trial (Tables 1 and 2).

The arithmetic mean TEQ concentrations of PCDDs, PCDFs, non-ortho PCBs, and mono-ortho PCBs in the blood of the 26 Yusho patients were 24, 82, 21, and $3.2 \mathrm{pg}$ TEQ/g lipid, respectively, before the trial, and 24, 82, 22, and $3.1 \mathrm{pg} T E Q / g$ lipid, respectively, after the trial. Total TEQ concentration of these dioxin-like compounds equaled 42-303 (mean: 130, median: 120) pg TEQ/g lipid before the trial, and 43-283 (mean: 132, median: 118) pg TEQ/g lipid after the trial, indicating that the concentrations before the trial were almost the same as those after the trial. Regarding the non-dioxinlike PCB concentrations, the sums of the concentrations of $58 \mathrm{PCB}$ congeners in the blood before and after the trial were 321-2643 (mean: 957, median: 872) and 286-2007 (mean: 975, median: 806) ng/g lipid, respectively. The arithmetic mean concentrations of triCBs, tetraCBs, 
pentaCBs, hexaCBs, heptaCBs, octaCBs, and nonaCBs in the blood of Yusho patients were 1.8, 20, 31, 402, 414, 79, and $7.8 \mathrm{ng} / \mathrm{g}$ lipid, respectively, before the trial, and 1.9, $20,33,412,420,79$, and $7.6 \mathrm{ng} / \mathrm{g}$ lipid, respectively, after the trial, indicating that concentrations of these PCBs compounds were also almost the same before and after the trial. These results indicated that the concentrations of PCDDs, PCDFs, dioxin-like PCBs and nondioxin-like PCBs in the blood of Yusho patients were not significantly altered by the intervention with oral colestimide.

We previously reported that the concentrations of 1,2,3,6,7,8-hexaCDD, 2,3,4,7,8-pentaCDF, 1,2,3,4,7,8-hexaCDF, 1,2,3,6,7,8-hexaCDF, hexaCB-169, hexaCB-156, hexaCB-157, and heptaCB-189 in the blood of Yusho patients were higher than those of the normal controls $[8,9]$. These can be considered the characteristic congeners in the blood of Yusho patients. 2,3,4,7,8-PentaCDF is recognized as the most important causative agent for subjective symptoms of Yusho. Blood levels before and after the trial were $48-636$ (mean: 241, median: 191) and 49-613 (mean: 242, median: 205) pg TEQ/g lipid, respectively, indicating that the concentration did not significantly decrease with administration of colestimide. This was also the case for the concentrations of other characteristic congeners before and after the trial. Among congeners of PCDDs, PCDFs, dioxin-like PCBs, and non-dioxin-like PCBs, most congeners did not show statistically significant differences. According to these results, the therapeutic usefulness of colestimide in reducing the concentrations of PCDDs, PCDFs, and PCBs in blood of Yusho patients could not be confirmed.

\section{Discussion}

Over 48 years have passed since the outbreak of Yusho disease. However, some patients are still afflicted with intractable symptoms such as chloracne, general fatigue and neuropathy [12]. There are patients who continue to have much higher concentrations of dioxin-like compounds in their blood than unaffected persons. Moreover, the half-lives of blood concentrations of 2,3,4,7,8-pentaCDF have become long to near infinity in the majority of Yusho patients [24]. To reduce the concentrations of PCDDs, PCDFs, and PCBs in the blood of Yusho patients, our study group previously conducted a clinical trial using cholestyramine and rice bran fiber $[19,20]$. Results of that study showed that the amounts of 2,3,4,7,8-pentaCDF in patients' feces actually increased, although beneficial clinical effects were not apparent, possibly due to a short trial period. A recent study reported that colestimide can decrease the concentrations of PCDDs, PCDFs, and PCBs in blood [21, 22]. Eight male and two female healthy subjects were treated with colestimide ( $3 \mathrm{~g} /$ day) for 6 months. In this report, colestimide was effective for promoting excretion of dioxin-like compounds from the human body. Colestimide is a non-absorbable anion exchange resin and enhances excretion of cholesterol in feces by inhibiting absorption of food-derived cholesterol in the intestinal tract [25]. Based on this result, we designed a clinical trial with colestimide for Yusho patients. However, in the present study, we were unable to confirm a significant decrease in most congeners of PCDDs, PCDFs, and PCBs in the blood of Yusho patients. It is suggested that the PCDDs, PCDFs, and PCBs that have remained in the whole body of patients over the 45 years since the outbreak of Yusho are very difficult to excrete from the body. In the present trial, there may be many limitations such as a small number of participants, duration of administration period and dose of cholestimide. Out of the 36 patients who participated in the trial, 9 patients experienced serious adverse effects (constipation or abdominal distension) by the repeated administration of colestimide. Therefore, we cannot recommend that elderly patients participate in clinical trial studies for such long periods as in the present study.

\section{Conclusion}

Although over 48 years have passed since the outbreak of Yusho, many patients still suffer various symptoms such as chloracne, general fatigue and neuropathy. The concentrations of causative dioxin-like compounds in their blood remain at high levels. We examined whether oral administration of colestimide could reduce the concentrations of PCDDs, PCDFs, and PCBs in the blood of Yusho patients. However, the effectiveness of colestimide on the concentrations of these dioxin-like compounds in the blood of Yusho patients could not be confirmed.

\section{Abbreviations \\ PCDDs, polychlorinated dibenzo-p-dioxins; PCDFs, polychlorinated dibenzofurans; PCBs, polychlorinated biphenyls; WHO, World health oganization; TEQ, toxic equivalent; TEF, toxic equivalency factor}

\section{Acknowledgements}

We would like to sincerely thank the participants to the study and all research staff at Research and Clinical Center for Yusho and Dioxin, Kyushu University Hospital.

\section{Funding}

This research was supported by a Grant-in-Aid for scientific research from the Ministry of Health, Labour and Welfare, Japan.

\section{Availability of data and materials}

We do not wish to share the data included in this manuscript. Patients who fulfilled the diagnostic criteria for Yusho established by the National Study Group for the Therapy of Yusho were eligible for this study. Therefore, we want to protect the patients' identities and personal information.

\section{Authors' contributions}

$\Pi$ developed the analytical method, and drafted the initial manuscript. AK, MI and $Y T$ examined the data quality for analyses. CM and MF interpreted the results. MF coordinated the project. All authors approved the final manuscript. 


\section{Competing interests}

The authors declare that they have no competing interests.

\section{Consent for publication}

Patients were recruited at explanatory meetings conducted in Fukuoka and Nagasaki Prefectures. 50 Yusho patients were enrolled in this clinical trial, and 36 patients participated in the trial. Informed consent was obtained for study participation. We also confirmed their consent for publication of this manuscript

\section{Ethics approval and consent to participate}

The study project was approved by the institutional ethics committee of Kyushu University Hospital (reference 18034).

\section{Author details}

${ }^{1}$ Kitakyushu Life Science Center, Public Interest Incorporated Foundation, Nakabarushinmachi 1-4, Tobata-ku, Kitakyushu-shi, Fukuoka 804-0003, Japan. ${ }^{2}$ Fukuoka Institute of Health and Environmental Sciences, 39, Mukaizano, Dazaifu-shi, Fukuoka 818-0135, Japan. ${ }^{3}$ Research and Clinical Center for Yusho and Dioxin, Kyushu University Hospital, Maidashi 3-1-1, Higashi-ku, Fukuoka 812-8582, Japan. ${ }^{4}$ Department of Dermatology, Graduate School of Medical Sciences, Kyushu University, Maidashi 3-1-1, Higashi-ku, Fukuoka 812-8582, Japan

\section{Received: 17 December 2015 Accepted: 30 May 2016}

\section{Published online: 04 June 2016}

\section{References}

1. Kuratsune M, Yoshimura H, Hori Y, Okumura Y, Masuda Y. Yusho. A human disaster caused by PCBs and related compounds. Fukuoka, Japan: Kyushu University Press; 1969.

2. Furue M, Uenotsuchi T, Urabe K, Ishikawa T, Kuwabara M. Overview of Yusho. J Dermatol Sci. 2005;1:S3-S10.

3. lida T, Todaka T. Measurement of dioxins in human blood: improvement of analytical method. Ind Health. 2003;41:197-204.

4. Todaka T, Hirakawa H, Tobiishi K, lida T. New protocol for dioxin analysis of human blood. Fukuoka Igaku Zasshi. 2003;94:148-57.

5. Todaka T, Hirakawa H, Hori T, Tobiishi K, lida T. Improvement in dioxin analysis of human blood and their concentrations in blood of Yusho patients. J Dermatol Sci. 2005;1:S21-S28.

6. Todaka T, Hirakawa H, Hori T, Tobiishi K, lida T. Follow-up survey of dioxins concentrations in the blood of Yusho patients in 2002-2003. Fukuoka Igaku Zasshi. 2005:96:249-58.

7. Todaka T, Hirakawa H, Kajiwara J, Hori T, Tobiishi K, Onozuka D, lida T, Yoshimira T, Furue M. Dioxin concentration in the blood of patients collected during medical check-up for Yusho in 2004-2005. Fukuoka Igaku Zasshi. 2007:98:222-31.

8. Todaka T, Hirakawa H, Hori T, Tobiishi K, lida T, Furue M. Concentrations of polychlorinated dibenzo-p-dioxins, polychlorinated dibenzofurans, and non-ortho and mono-ortho polychlorinated biphenyls in blood of Yusho patients. Chemosphere. 2007:66:1983-9.

9. Todaka T, Hori T, Hirakawa H, Kajiwara J, Yasutake D, Onozuka D, lida T, Furue M. Concentrations of polychlorinated biphenyls in blood of Yusho patients over 35 years after the incident. Chemosphere. 2009;74:902-9.

10. Todaka T, Hori T, Yasutake D, Yoshitomi H, Hirakawa H, Onozuka D, Kajiwara J, lida T, Yoshimura T, Furue M. Concentrations of polychlorinated biphenyls in blood collected from Yusho patients during medical check-ups performed from 2004-2007. Fukuoka Igaku Zasshi. 2009;100:156-65.

11. Imamura T, Kanagawa Y, Matsumoto S, Tajima B, Uenotsuchi T, Shibata S, Furue M. Relationship between clinical features and blood levels of pentachlorodibenzo-Furan in patients with Yusho. Environ Toxicol. 2007; 22:124-31.

12. Kanagawa Y, Matsumoto S, Koike S, Tajima B, Fukiwake N, Shibata S, Uchi H, Furue M, Imamura T. Association of clinical findings in Yusho patients with serum concentrations of polychlorinated biphenyls, polychlorinated quarterphenyls and 2,3,4,7,8-pentachlorodibenzofuran more than 30 years after the poisoning event. Environ Health. 2008;7:47.

13. Boylan JJ, Guzelian PS. Cholestyramine: Use as a new therapeutic approach for chlordecone (kepone) poisoning. Science. 1978;199:893-5.

14. Morita K, Matsueda T, lida T. Effect of dietary fiber on fecal excretion of polychlorinated dibenzo-p-dioxins in rats. Jpn J Toxicol Environ Health. 1997:43:35-41 (in Japanese).
15. Rozman T, Rozman K, Williams J, Greim H. Enhanced fecal excretion of mirex in rhesus monkeys by $5 \%$ mineral oil in the diet. Drug Chem Toxicol. 1981;4:251-62.

16. Rozman K, Rozman T, Greim H, Nieman IJ. Smith GS Use of aliphatic hydrocarbons in feed to decrease body burdens of lipophilic toxicants in livestock. J Agric Food Chem. 1982;30:98-100

17. Geusau A, Tschachler E, Meixmer M, Sandermann S, Päpke O, Wolf C, Valic E, Stingl G, McLachlan M. Olestra increases faecal excretion of 2,3,7,8-tetrachlorodibenzo-p-dioxin. Lancet. 1999:354:1266-7.

18. Moser GA, McLachlan MS. A non-absorbable dietary fat substitute enhances elimination of persistent lipophilic contaminants in humans. Chemosphere. 1999;39:1513-21.

19. lida T, Nakagawa R, Hirakawa H, Matsueda T, Morita K, Hamamura K, Hori Y, Guo Y, Chang F, Hsiao P, Lin K, Yu M, Lai T, Chen S, Hsu C. Clinical trial of a combination of rice bran fiber and cholestyramine for promotion of fecal excretion of retained polychlorinated dibenzofuran and polychlorinated biphenyl in Yu-Cheng patients. Fukuoka Igaku Zasshi. 1995;86:226-33.

20. Takenaka S, Morita K, Tokiwa H, Takahashi K. Effects of rice brain fiber and cholestyramine on the fecal excretion of Kanechlor 600 (PCB). Xenobiotica. 1991;16:1707-12.

21. Sakurai $K$, Todaka E, Saito $Y$, Mori C. Pilot study to reduce dioxins in the human body. Intern Med. 2004:43:792-5.

22. Sakurai K, Fukata H, Todaka E, Saito Y, Bujo H, Mori C. Colestimide reduces blood polychlorinated biphenyls (PCB) levels. Intern Med. 2006:45:327-8.

23. Van den Berg M, Birnbaum L, Denison M, De Vito M, Farland W, Feeley M, Fiedler H, Hakansson H, Hanberg A, Haws L, Rose M, Safe S, Schrenk D, Tohyama C, Tritscher A, Tuomisto J, Tysklind M, Walker N, Peterson RE. The 2005 World Health Organization re-evaluation of human and mammalian toxic equivalency factors for dioxins and dioxin-like compounds. Tox Sci. 2006;93:223-41.

24. Matsumoto S, Akahane M, Kanagawa Y, Kajiwara J, Mitoma C, Uchi H, Furue M, Imamura T. Unexpectedly long half-lives of blood 2,3,4,7,8pentachlorodibenzofuran (PeCDF) levels in Yusho patients. Environ Health. 2015; doi: 10.1186/s12940-015-0059-y.

25. Kishimoto N, Fujii S, Chiba H, Sakuma I, Tsutsui H. Colestimide, an anion exchange resin agent, can decrease the number of LDL particles without affecting their size in patients with hyperlipidemia. J Cardiol. 2010:55:65-8.

\section{Submit your next manuscript to BioMed Central} and we will help you at every step:

- We accept pre-submission inquiries

- Our selector tool helps you to find the most relevant journal

- We provide round the clock customer support

- Convenient online submission

- Thorough peer review

- Inclusion in PubMed and all major indexing services

- Maximum visibility for your research

Submit your manuscript at www biomedcentral.com/submit
C Biomed Central 